\title{
Convex Central Configurations of Two Twisted $n$-gons
}

\author{
E. Barrabés and J.M. Cors \\ Departament d'Informàtica i Matemàtica Aplicada, \\ Universitat de Girona \\ barrabes@imae.udg.edu \\ Departament de Matemàtica Aplicada III, \\ Universitat Politècnica de Catalunya \\ cors@epsem.upc.edu
}

\section{Introduction}

The simplest motions that can be found in the Newtonian $N$-body problem are the ones such that the configuration is constant up to rotations and scaling, and every body follows a trajectory that is a keplerian orbit. Such kind of solutions are called "central configurations".

We consider the planar $2 n$ body problem, where the masses are located at the vertices of two regular $n$-gons, $n \geq 2$, and all the masses at the same $n$-gon are equal, $m_{1}$ and $m_{2}$. In [3], Moeckel and Simó consider the case of two nested regular $n$-gons, that is, when the vertices of the two gons are aligned. They prove that for all values of $n$ and every ratio $m_{1} / m_{2}$, there are exactly two planar central configurations. In [2], the authors study the case of two twisted $n$-gons, where one of the two gons is rotated an angle of $\pi / n$ with respect the other. In that case, the authors prove that the number of central configurations depends on $n$.

Several authors have studied the convex central configurations in the four body problem. A classical result due to MacMillan and Bartky (Trans. Amer. Math. Soc. 34 (1932)) states that, for any four positive masses and any assigned order, there exists a convex planar central configuration. Xia in [6] gives a simple proof of that case. Albouy et al, in $[\mathbf{1}]$, prove that in the planar four-body problem, a convex central configuration is symmetric with respect to one diagonal if and only if the masses of the two particles on the other diagonal are equal. As fas as we know, it is a conjecture that given four masses in a certain order, there exists only one convex central configuration.

We present here some results concerning to central configurations of two twisted $n$-gons that are convex.

\section{Equations}

We consider two groups of $n$ bodies in the same plane $(x, y)$ at positions $\mathbf{q}_{j i} \in \mathbb{R}^{2}$, $i=1, \ldots, n, j=1,2$. All the bodies in the same group have equal mass, $m_{1}$ and $m_{2}$, respectively. It is well known (see [5]) that a central configuration (CC) of the planar $2 n$-body problem is a solution $\mathbf{q}=\left(\mathbf{q}_{11}, \mathbf{q}_{12}, \ldots, \mathbf{q}_{2 n}\right) \in \mathbb{R}^{4 n}$ of the equation

$$
\nabla U(\mathbf{q})+w^{2} M \mathbf{q}=0,
$$


for some value of $w$, where $U$ is the Newtonian potential

$$
U(\mathbf{q})=\sum_{j=1}^{2} \sum_{i=1}^{n}\left(\sum_{l=i+1}^{n} \frac{m_{j}^{2}}{\left\|\mathbf{q}_{j i}-\mathbf{q}_{j l}\right\|}+\sum_{l=j+1}^{n} \sum_{m=1}^{n} \frac{m_{j} m_{l}}{\left\|\mathbf{q}_{j i}-\mathbf{q}_{l m}\right\|}\right),
$$

and $M$ is the diagonal mass matrix with diagonal $m_{1}, \ldots, m_{1}, m_{2}, \ldots, m_{2}$ (each mass repeated $n$ times).

We consider CC consisting in two regular $n$-gons rotated an angle $\pi / n$ with respect each other. Without loss of generality, we can think that the first gon has one vertex on the positive horizontal axis, $y=0, x>0$, and it is contained in a circle of radius 1 .

Introducing $\mathbf{q}_{1 k}=e^{i \pi 2 k / n}$ and $\mathbf{q}_{2 k}=a e^{i \pi(2 k+1) / n}$, for $k=0, \ldots, n-1$ and $a$ is the radius of the circle that contain the second gon (that can be viewed as the size of the second $n$-gon), then, the system of equations (1) can be written as $4 n$ real equations: $2 n$ corresponding to the real parts and $2 n$ to the imaginary parts. Due to the symmetries, the $2 n$ equations corresponding to the imaginary parts vanish (in fact, the only two possibilities for two gons to be in a CC are being nested or twisted, see [7]), and the $2 n$ equations corresponding to the real parts reduce to only 2. Finally, eliminating the variable $w$, the equations for the $\mathrm{CC}$ reduce to the following single equation

$$
\left(C_{2}(a)-S_{n} a\right) m_{1}+\left(\frac{S_{n}}{a^{2}}-a C_{1}(a)\right) m_{2}=0,
$$

where $S_{n}=\frac{1}{4} \sum_{k=1}^{n-1} \frac{1}{\sin (k \pi / n)}$, and the coefficients $C_{j}(a)$ are

$$
\begin{aligned}
& C_{1}(a)=\sum_{k=1}^{n} \frac{1-a \cos ((2 k-1) \pi / n)}{\left(1+a^{2}-2 a \cos ((2 k-1) \pi / n)\right)^{3 / 2}}, \\
& C_{2}(a)=\sum_{k=1}^{n} \frac{a-\cos ((2 k-1) \pi / n)}{\left(1+a^{2}-2 a \cos ((2 k-1) \pi / n)\right)^{3 / 2}} .
\end{aligned}
$$

We say that a value of $a$ is admissible if there exists a solution of (2) with $m_{1}>0$ and $m_{2}>0$. In $[\mathbf{2}]$ the admissible values of $a$ and the number of CC for a given mass ratio $m_{2} / m_{1}$ are studied.

The equation (2) was also obtained by Roberts in [4] and Yu and Zhang in [7], and it is similar to the one obtained by Simó and Moeckel in [3] for the nested case.

\section{Convex CC of two twisted $n$-gons}

Given an admissible value of $a$, we discuss here whether the configuration is convex or not. It is not difficult to see that, given $2 n$ bodies distributed uniformly in two twisted regular $n$-gons, the configuration is convex if and only if

$$
\cos \left(\frac{\pi}{n}\right) \leq a \leq \frac{1}{\cos \left(\frac{\pi}{n}\right)}
$$

We will present the results separately for $n=2,3,4$ and $n \geq 5$.

The case $n=2$, that is two bodies in each group, can be summarized in the following result proved by MacMillan and Bartky.

Theorem 1 For any positive value of the mass ratio $m_{2} / m_{1}$, there exists only one $C C$ of two twisted segments and it is convex. 
We will present the results considering values of the mass ratio $m_{2} / m_{1} \geq 1$. By reescaling, the number of convex central configurations for $m_{2} / m_{1}<1$ is obtained.

In the case $n=3$ (two equilateral triangles rotated $\pi / 3$ ), we prove the following result:

Theorem 2 Consider 6 masses located at the vertices of two twisted equilateral triangles, such that all the masses in one triangle are equal to $m_{1}$ and the masses of the other triangle are equal to $m_{2}$. Then, there exists one value for the mass ratio $m_{2} / m_{1}, m^{*}>1$ such that

(1) for any $m_{2} / m_{1} \in\left(1, m^{*}\right)$, there exist exactly three different convex central configurations,

(2) for any $m_{2} / m_{1} \in\left(m^{*}, \infty\right)$ there exists only one convex central configuration,

(3) for $m_{2} / m_{1}=1, m^{*}$ the number of different convex central configurations is exactly two.

The approximate value of $m^{*}=1.0007682$. When $m_{2}=m_{1}$, the two convex central configurations where already numerically computed by Moeckel in an unpublished report. In Figure 1 we show three examples of convex CC in the case of two twisted equilateral triangles.
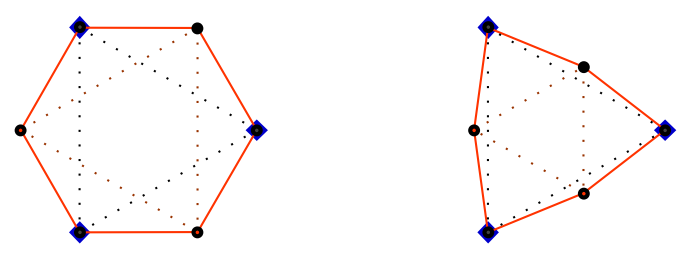

Figure 1. Convex CC of two twisted equilateral triangles corresponding to $m_{2}=m_{1}$ (left, the regular hexagon) and the limit case $m_{2} / m_{1}=\infty$ (right)

All the results are based on the number of solutions of equation (2) that correspond to the mass ratio $m_{2} / m_{1}=0$. For $n=3$ it can be shown that there exists exactly two of that solutions, but in the case of $n \geq 4$, it can be shown that there exists two, but as far as we know, it is not proved that they are the only ones. The results for $n \geq 4$ rely on the truthfulness of the following conjecture.

\section{Conjecture}

For $n \geq 4$ there exists exactly two solutions of the equation (2) that correspond to $m_{2}=0$ and $m_{1} \neq 0$.

Under that assumption, it can be shown the following results:

Theorem 3 Consider 8 masses located at the vertices of two twisted squares, such that all the masses in one square are equal to $m_{1}$ and the masses of the other square are equal to $m_{2}$. There exists a value for the mass ratio $m_{2} / m_{1}=\bar{m}$ such that

(1) for any $m_{2} / m_{1} \in[1, \bar{m}]$, there exists exactly one convex central configuration,

(2) for any $m_{2} / m_{1} \in(\bar{m}, \infty)$ there exists no convex central configuration.

The approximate value of $\bar{m}=16.05679941$. 
Theorem 4 Consider $2 n$ masses, with $n \geq 5$, located at the vertices of two $n$-gons, such that all the masses in one gon are equal to $m_{1}$ and the masses of the other gon are equal to $m_{2}$. For any value of the mass ratio $m_{2} / m_{1}$, there exists at least one convex central configuration.

In summary, we observe that always exists a convex $\mathrm{CC}$ of twisted rings for any value of $n$, except for $n=4$ ( 8 body problem). Moreover, the richer case is $n=3$ where there exists values of the mass ratio with three convex CC.

\section{References}

[1] A. Albouy, Y. Fu, S. Sun. Symmetry of Planar Four-Body Convex Central Configurations Proc. R. Soc. A 464(2093): 1355-1365, 2008

[2] E. Barrabés, J.M. Cors, G. Roberts. Planar Central Configurations of Twisted Rings. Preprint

[3] R. Moeckel and C. Simó. Bifurcation of spatial central configurations from planar ones. SIAM journal on mathematical analysis, 26:978-998, 1995.

[4] Gareth E. Roberts. Existence and Stability of Relative Equilibria in the N-Body Problem. PhD thesis, Boston University, 1999.

[5] D. G. Saari. Collisions, rings, and other Newtonian N-body problems, volume 104 of CBMS Regional Conference Series in Mathematics. Published for the Conference Board of the Mathematical Sciences, Washington, DC, 2005.

[6] Z. Xia. Convex central configurations for the n-body problem Journal of Differential Equations, 200 (2): 185-190, 2004

[7] X. Yu and S. Zhang. Twisted angles for central configurations formed by two twisted regular polygons J. Differential Equations 253:2106-2122, 2012. 